\title{
Late pulmonary metastasis of inflammatory breast cancer 26 years after initial radiation therapy alone
}

\author{
Kun-Ming $\mathrm{Wu}^{1,2}$, Chih-Hao Chen ${ }^{* 3,4,5}$, Ho Chang ${ }^{3}$, Chao-Hung Chen ${ }^{4,5}$ \\ ${ }^{1}$ Mackay Medicine, Nursing and Management College, Taipei City, Taiwan \\ ${ }^{2}$ Chest Division, Department of Internal Medicine, Mackay Memorial Hospital, Taipei City, Taiwan \\ ${ }^{3}$ Graduate Institute of Manufacturing Technology, National Taipei University of Technology, Taipei City, Taiwan \\ ${ }^{4}$ 4Department of Thoracic Surgery, Mackay Memorial Hospital, Taipei City, Taiwan \\ ${ }^{5}$ Department of Medicine, Mackay Medical college, Taipei City, Taiwan
}

Received: November 2, 2015

DOI: $10.5430 /$ css.v2n2p40
Accepted: March 1, 2016

Online Published: March 14, 2016

URL: http://dx.doi.org/10.5430/css.v2n2p40

\begin{abstract}
A 64-year-old woman underwent cobalt-60 radiation therapy 26 years ago because of advanced inflammatory breast cancer. The initial stage was T4N0M0. During the course of cobalt-60 therapy, the patient presented severe chest wall necrosis. Her chest wall wound healed very slowly and she decided to stop radiation therapy and declined both surgery as well as chemotherapy. After chronic wound healing, the patient did not come back for follow-up. Twenty-six years later, she came back for mild dyspnea. Subsequent study showed suspicious lung metastasis. Later, she underwent thoracoscopic biopsy, with which lung metastasis was confirmed. She recovered well and currently receives oral chemotherapy for disease control.
\end{abstract}

Key Words: Metastasis, Breast cancer, Thoracoscopy/ Video-assisted thoracoscopic surgery

\section{INTRODUCTION}

Current management of breast cancer is multidisciplinary, including effective resection of breast cancer tissues and chemoradiation depending on the involved margin, nodal status and final pathologic stage. Radiation alone was not an effective single strategy to treat breast cancer. It was considered as a method of local control and will not result in better long-term survival. In the case, we reported an unusual condition. She did not receive any form of treatment except for incomplete cobalt-60 radiation therapy. During the 26-year period, she did not come back for follow-up and stated to be healthy without any discomfort.

\section{CASE REPORT}

A 64-year-old woman was found to have inflammatory breast cancer, locally advanced in right side peri-areolar tissues 26 years ago when she was 38 years old. Because the initial condition was quite severe, the initial treatment plan was preoperative radiation therapy followed by surgical resection. She underwent surgical biopsy of the lesion and confirmed it to be infiltrating ductal carcinoma. Bone scan and other survey showed that there was no evidence of distant metastasis. The planned cobalt- 60 radiation was daily $180 \mathrm{cGy}$ for a total does of $5400 \mathrm{cGy}$. The covered areas included right side chest, right half of the neck, right side axillary and the sternum. After accumulated dose of $4680 \mathrm{cGy}$, the patient complained of marked chest wall pain. Inspection showed erythematous and necrosis of the skin and soft tissues in right side parasternal region. Then the cobalt therapy was held. The erythematous skin and soft tissues became progressively ruptured and necrotic. The patient experienced progressive chest wall necrosis after radiation. The patient then went

\footnotetext{
*Correspondence: Flávio L. C. Pádua; Email: cardeal@decom.cefetmg.br; Address: Departamento de Computacao, Centro Federal de Educação Tecnológica de Minas Gerais, Av. Amazonas, 7675, Zip Code: 30.510-000, Belo Horizonte, Brazil.
} 
home for self-care of the wound. But the patient did not come back anymore. One and a half year, she came back for wound evaluation. We found that her chest wall wound was dry with obvious scar after secondary healing. However, she declined to undergo surgical intervention. During the subsequent 24.5 years, she did not come back for follow-up. This time, she sought medical attention again because she complained of mild dyspnea. Chest radiograph showed pleural effusions of small amount. Very faint opacity can be seen in middle to lower lung fields(see Figure 1A). Computed tomographic scan (CT scan) showed some soft lesions in right middle lobe in both soft tissue window and lung window (see Figure 1B and 1C). Other cancer survey excluded the possibility of extra-pulmonary origin. Her left side breast was free from any lesion based on gross palpation, CT scan and mammography. Her chest wall on admission showed an old and dense scar in the right half. Palpation is dry and fibrotic (see Figure 2). There was no palpable mass. Nipple discharge was absent. Because the patient's clinical manifestations were not typical of pneumonia. Malignancy was still one possibility. Responses to empiric antibiotics were limited. Therefore, we had to confirm the nature of the pulmonary lesions. We then prepared for thoracoscopic biopsy of the lung and pleura. The approach is single port approach with an incision around $1.5 \mathrm{~cm}$. On entering the pleural space, there were some clear effusions, which were aspirated for cytology. The lung was found to have dense adhesion in some parts (see Figure 3A). Close observation showed some whitish spots and marked anthracosis (see Figure 3B and 3C). In some visceral pleural surfaces, scattered whitish nodulations were seen (see Figure 3D). Wedge resection of the lesions showed metastatic carcinoma on frozen section. The final pathology report showed metastatic adenocarcinoma which was positive for estrogen receptor and progesterone receptor. The cells were negative for TTF-1.The effusion was negative for cancer cells. The chest tube was removed 48 hours after the operation. The patient still declined any form of intravenous chemotherapy. Instead, she was on oral chemotherapy now. Currently, she was followed up in the outpatient department of radiation oncologist.

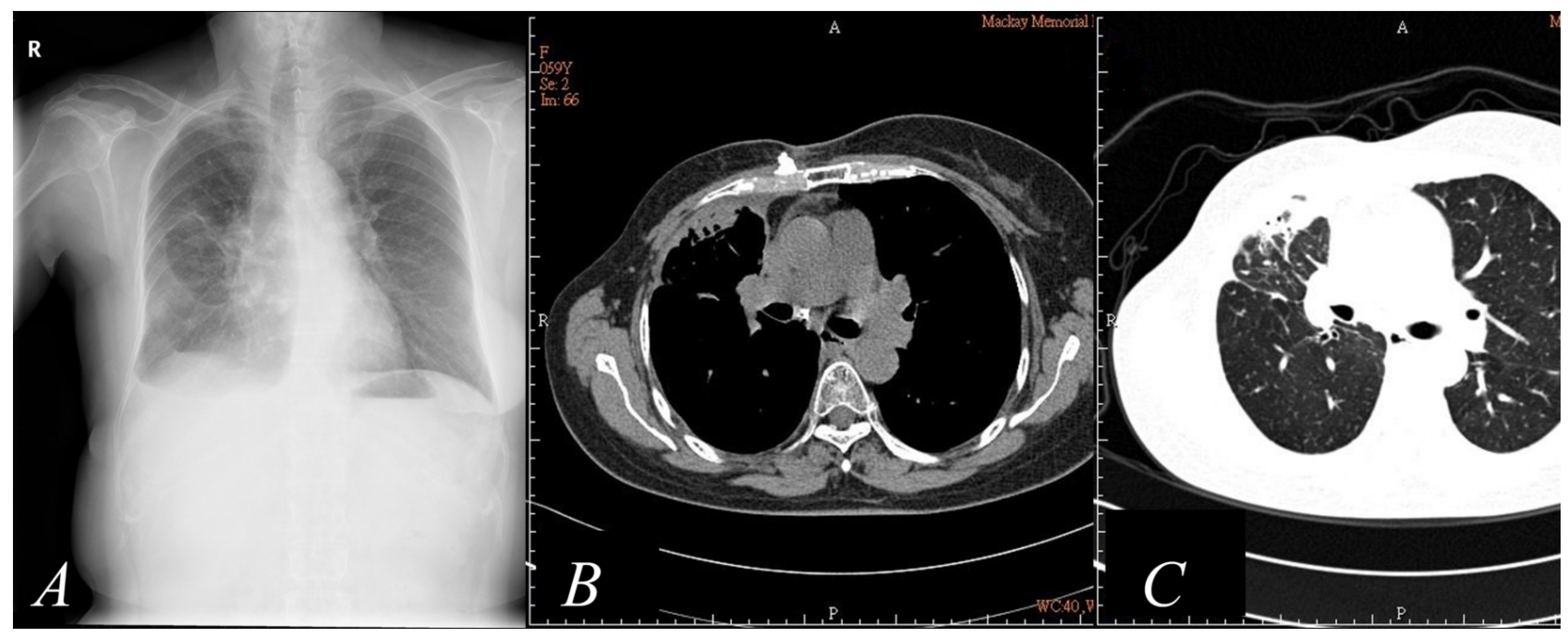

Figure 1. Image study of the patient

(A) Her chest radiograph showed some pleural effusions in right side and faint opacity in middle to lower lung fields; (B) CT scan in soft tissue window showed atrophic breast tissues and lesions in right lung as well as parietal pleura; Calcification in the chest wall was also seen beneath prior wound; (C) Lung window showed some irregular lesions in right middle lobe

\section{Discussion}

The standard treatment of breast cancer requires multidisciplinary strategies, including effective resection with or without chemoradiation. ${ }^{[1]}$ The role of radiation was thought to be local disease control. Radiation therapy would not cure the disease. But it remained unclear that how long can radiation therapy alone to control the disease. ${ }^{[2]}$ In the reported case, the patient's breast cancer was suppressed locally for

Published by Sciedu Press very long period without distant metastasis. Although with initial skin necrosis and poor healing, the patient did not experience any physical abnormality during the following 25 years.

Even with standard treatment, the recurrence rate of breast cancer was around $4 \%$ after ten years. Although the recurrence rate declines very slowly but recurrence after 20 years is still quite rare. ${ }^{[3,4]}$ Recurrence 26 years later after an initial 
radiation therapy alone had not bee reported.

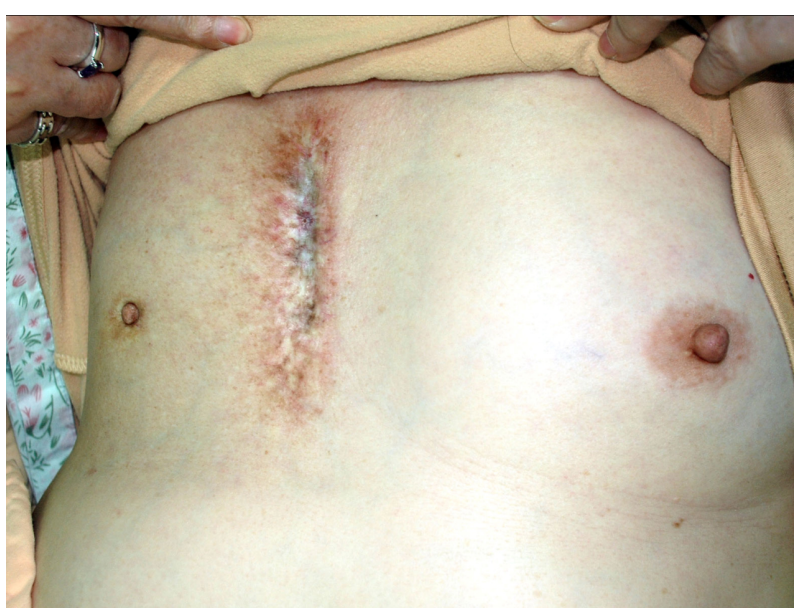

Figure 2. Gross appearance of the chest wall after radiation therapy

Her right side chest wall was necrotic at that time she received cobalt-60 therapy. She later experienced prolonged course of wound care for nearly one year with the end result of an atrophic soft tissues and radiation scar near the parasternal region. Her left side breast was grossly and radiographically normal.
In the past, cobalt-60 therapy was the sole option of radiation and the three-dimensional planning was inaccurate compared to that of current stereotactic radiation therapy. However, with adequate coverage and effective dose, breast cancer could be controlled for long period. In patients with locally advanced breast cancer but with poor performance, accurate coverage with effective accumulated dose of radiation may be considered as a plausible alternative.

In view of clinical presentation and her CT scan, the patient's manifestations were not typical as in treatment failure. Treatment failure can be either local recurrence of distant metastasis. In the patient's condition, the image features of metastatic lesions were not typical on CT scan and may confuse us for proper decision of surgical biopsy. Since initial radiologist described her CT scan as inflammatory and fibrotic changes, they preferred the diagnosis of radiation pneumonitis. CT-guided biopsy was declined by them. Because malignancy in the lung can't be excluded by image study alone, thoracoscopic biopsy is mandatory for definite diagnosis, which is important to guide subsequent treatment plan.

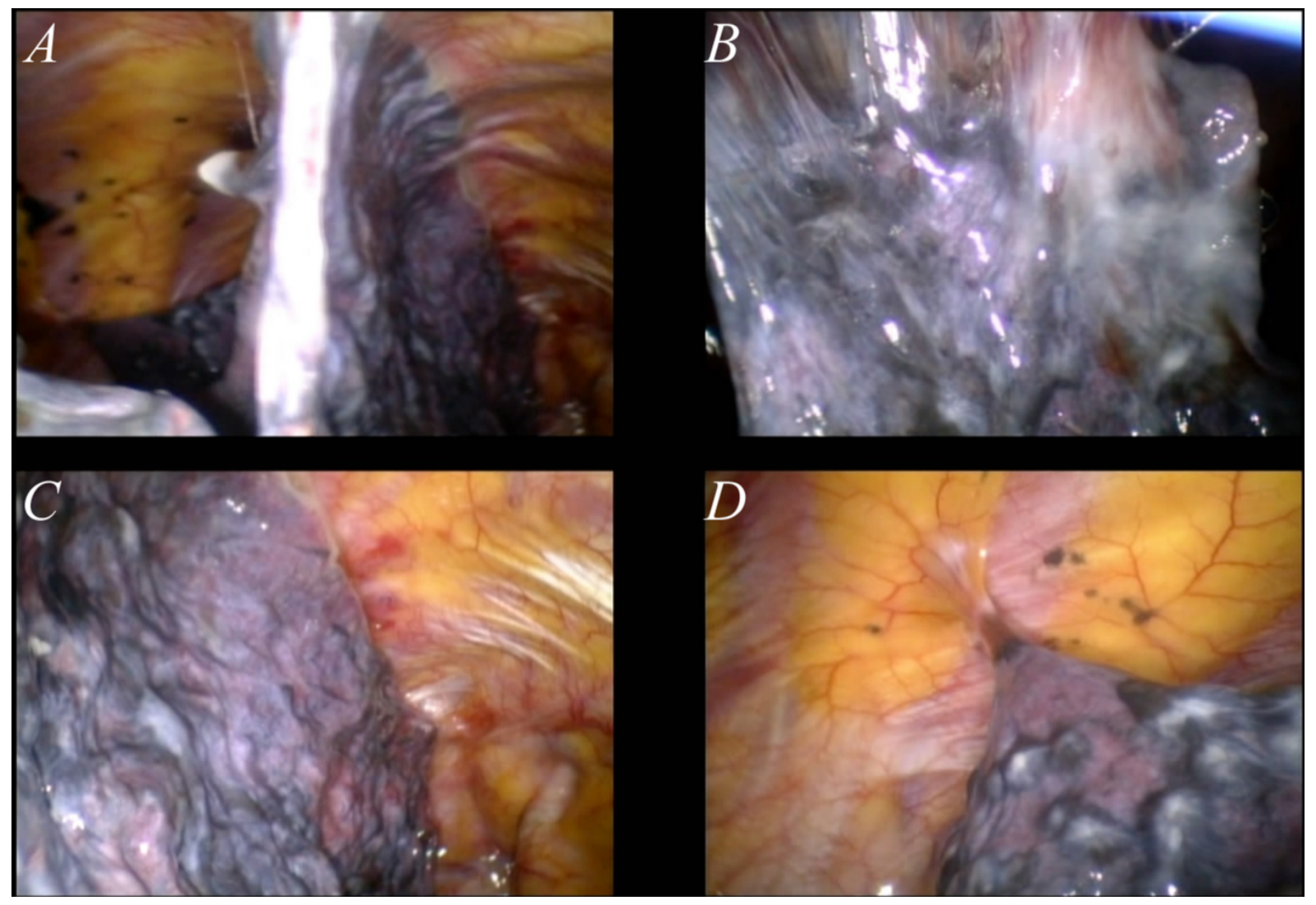

Figure 3. Thoracoscopic views of the lung and pleura

(A) On entering the pleural space, the middle lung was found to be densely adhered in the chest wall; (B) Adhesion bands and whitish fibrotic changes were seen clearly in the middle lobe; and $(C)$ inferior margin of upper lobe; $(D)$ Some whitish nodulations with lung tissues were resected for biopsy 


\section{Conclusions}

The reported case raised two important issues. The first issue is the complexity of tumor biology. Even after protracted period, recurrence or metastasis may still occur. The cells could be so indolent after local radiation alone. In patients with prior history of breast cancer, recurrence or metastasis is still possible after decades. The second issue is that aggres- sive surgical biopsy is essential for such condition. When clinical decision making is difficult, surgical biopsy may be an important step for definite diagnosis and guide subsequent treatment.

\section{CONFLicts OF INTEREST Disclosure}

The authors declare they have no conflict of interest.

\section{REFERENCES}

[1] Roberto P, Manuel C, Laura C, et al. Radiation therapy following surgery for localized breast cancer: outcome prediction by classical prognostic factors and approximated genetic subtypes. Journal of Radiation Research. 2013; 54(2): 292-298. PMid: 23019151. http://dx.doi.org/10.1093/jrr/rrs087

[2] Jonathan YT, Ho AY. Radiation therapy in the management of breast cancer. Surg Clin North Am. 2013; 93(2): 455-71. PMid: 23464696. http://dx.doi.org/10.1016/j.suc. 2013.01.002

[3] Héquet D, Bricou A, Koual M, et al. Systematic cavity shaving: mod- ifications of breast cancer management and long-term local recurrence, a multicentre study. European Journal of Surgical Oncology the Journal of the European Society of Surgical Oncology \& the British Association of Surgical Oncology. 2013; 39(8): 899-905. http://dx.doi.org/10.1016/j.ejso.2013.05.012

[4] Sangen MJCVD, Scheepers SWM, Poortmans PMP, et al. Detection of local recurrence following breast-conserving treatment in young women with early breast cancer: Optimization of long-term follow-up strategies. Breast. 2013; 22(3): 351-356. PMid: 22989668. http://dx.doi.org/10.1016/j.breast. 2012.08.006 